\title{
„Die moderne Medizin ist aufgrund ihrer Komplexität nur noch im interdisziplinären Austausch möglich.“
}

Pathologie und Radiologie mit ihren bildgebenden Verfahren durchlaufen viele kongruente Entwicklungen und stehen vor ähnlichen Herausforderungen wie beispielsweise der zunehmenden fachlichen Komplexität oder der wachsenden Digitalisierung der Arbeitsprozesse. Verbesserte Techniken und die stete Optimierung der Diagnostik bieten wiederum neue Möglichkeiten der Zusammenarbeit. Um den Austausch zwischen Pathologen und Radiologen zu ermöglichen und Grundkenntnisse des jeweils anderen Fachs zu vermitteln, kooperieren die Deutschen Gesellschaften für Pathologie und Radiologie in vielfältiger Art und Weise. Prof. Dr. Ruth Knüchel-Clarke, Direktorin des Instituts für Pathologie an der Uniklinik RWTH Aachen und Prof. Dr. Thorsten Bley, Direktor des Instituts für Röntgendiagnostik am Universitätsklinikum Würzburg werfen gemeinsam einen Blick auf die beiden Fachdisziplinen.

? Frau Professor Knüchel-Clarke, Ende Mai fand die 100. Jahrestagung der Deutschen Gesellschaft für Pathologie in Berlin statt. War dies für Sie als Kongresspräsidentin ein Anlass, den Blick zurück zu werfen, oder haben Sie damit vielmehr den Ausgangspunkt für neue Perspektiven und Herausforderungen verbunden?

Knüchel-Clarke: Einen Blick zurück zu werfen ist für meine Generation, aber auch für jüngere Kollegen äußerst wertvoll. In der Geschäftigkeit des Alltags bleibt zu wenig Zeit zur Reflexion; das Jubiläum hat deshalb auch den Raum dafür geöffnet, Lernmöglichkeiten aus der Rückschau zu generieren. Neue Perspektiven und Herausforderungen aufzuzeigen, sollte Inhalt eines jeden guten Kongresses sein und stand deshalb auch bei der 100 . Jahrestagung im Mittelpunkt. Diese liegen in der Pathologie bekanntermaßen in der Molekularpathologie. Zusätzlich wollten wir diesmal aber auch die Entwicklungen in der Bildgebung im konstruktiven Austausch mit der Radiologie und Nuklearmedizin bekannt machen. In der Uropa-

thologie, meinem eigenen Spezialgebiet, haben neue Forschungsergebnisse erst jüngst zur Neuauflage des WHO-Buchs über urologische Tumoren und zur Aktualisierung verschiedener uroonkologischer Leitlinien geführt. Entsprechend war auch dies ein zentrales Thema von Fortbildungsveranstaltungen und wissenschaftlichen Diskussionen.

\section{? Herr Professor Bley, wo sehen Sie Parallelen, wenn Sie die beiden Fachdisziplinen Pathologie und Ra- diologie miteinander vergleichen?}

Bley: Beide Fachdisziplinen, die Pathologie und die Radiologie, sind bildgebende Verfahren. In der Radiologie werden anhand von radiografischen, CT-grafischen, MR-grafischen oder sonografischen Bildeindrücken, in der Pathologie anhand von makroskopischen und mikroskopischen Präparaten Diagnosen gestellt. In der Radiologie wird hierfür die spezifische und je nach Entität unterschiedliche Kontrastmittelanreicherung der verschiedenen Gewebe zurate gezogen. Die Pathologie wiederum bedient sich vielfältiger Verfahren wie zum Beispiel der Immunhistochemie und molekularpathologischer Techniken, um die histologischen Diagnosen weiter zu spezifizieren. In beiden Disziplinen ist die exakte Kenntnis des jeweiligen klinischen Kontextes notwendig, um präzise Diagnosen stellen zu können.

? Beide Disziplinen sind mit einer immer größeren Komplexität und einer zunehmenden Subspezialisierung konfrontiert. Erkennen Sie hier eine ernstzunehmende Bedrohung für die Einheit der Pathologie bzw. der Radiologie?

Knüchel-Clarke: Für einen Arzt - und ein Pathologe ist auch ein Arzt - besteht das höchste Ziel in der bestmöglichen Versorgung seiner Patienten. Diesem Ziel sind die Fächerrollen absolut nachgeordnet. Die moderne Medizin ist aufgrund ihrer Komplexität nur noch im interdisziplinären Austausch möglich. Und dieser Aus- tausch wird im Interesse des Patienten bereits in vielfältiger Form, zum Beispiel durch interdisziplinäre Konferenzen, praktiziert. Ich bin der Überzeugung, dass bei möglichst sachlicher Wertung der Fähigkeiten der verschiedenen Fächer jeder auch dann genug zu tun hätte, wenn sich Fächergrenzen verschieben würden. Die Subspezialisierung der Pathologie erfolgt bereits und sollte auch weiterhin durch Netzwerke zwischen Pathologien und der Festlegung von Referenzzentren ermöglicht werden. Technische Entwicklungen wie die digitale Mikroskopie und neue Formen gemeinsamer Datenbanken und Biobanken helfen, auch seltenere Fälle zeitnah zu lösen. Unterstützt durch diese Netzwerke werden die akademischen Pathologien auch weiterhin in der Lage sein, den holistischen Anspruch der Lehre patho-physiologischer Prozesse mittels der Morphologie als zentrales Element der Medizinerausbildung zu vermitteln.

Bley: Ähnliches gilt auch für die Radiologie. In den interdisziplinären Konferenzen wird auf Augenhöhe mit den zum Teil sehr stark spezialisierten klinischen Fächern diskutiert. Um dieses Niveau in unseren Befunden und in den interdisziplinären Konferenzen auch in Zukunft gewährleisten zu können, ist eine Spezialisierung innerhalb des großen Querschnittfachs Radiologie notwendig. Die Zusatzqualifikationen in der Kinderradiologie und Neuroradiologie haben in der Vergangenheit schon gezeigt, wie wichtig eine vertiefte Weiterbildung in diesen Spezialgebieten ist. Weitere Bemühungen zur Spezialisierung spiegeln sich in den zahlreichen $\mathrm{Ar}$ beitsgemeinschaften innerhalb der Deutschen Röntgengesellschaft wider. Wichtig dabei ist, dass der von Frau Knüchel-Clarke angesprochene Zusammenhalt des Fachgebiets nicht aufgebrochen wird, sondern die möglichen Synergieeffekte zum Vorteil der einzelnen Spezialisierungen realisiert werden. Darüber hinaus wachsen die Fachgebiete der Nuklearmedizin und der Radiologie etwa im Bereich

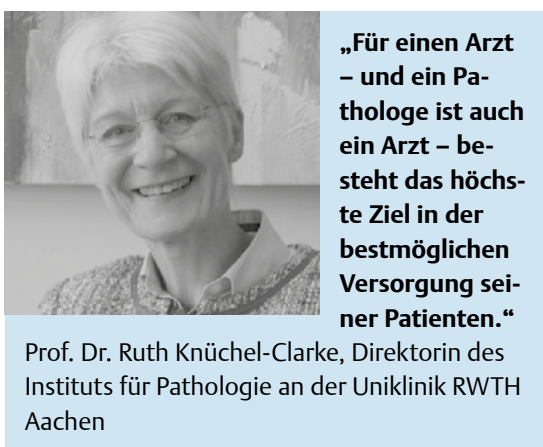


der Hybrid-Bildgebung PET/CT im klinischen Alltag weiter zusammen. In Würzburg befunden Radiologen und Nuklearmediziner Seite an Seite gemeinsam die PET/CT-Untersuchungen, um die bestmögliche diagnostische Genauigkeit durch die Expertise aus beiden Blickwinkeln zu erlangen.

? Wie bewerten Sie vor Ihrem fachlichen Hintergrund die voranschreitende Digitalisierung der Arbeitsprozesse bzw. welche Veränderungen erleben Sie bereits in Ihrem Arbeitsalltag?

Knüichel-Clarke: Ich denke, die Pathologie nähert sich in den Prozessen der Radiologie an und kann hier sicherlich viel von ihr lernen. Die Entwicklungen der Speicherkapazitäten im IT-Bereich machen die Möglichkeit immer realistischer, das eine Mikroskopie am Computer trotz hoher Datenmengen wegen notwendiger Farbdarstellung nutzbar ist. Hierbei spreche ich vom Einscannen von histochemisch oder immunhistochemisch gefärbten Objektträgern und - ähnlich dem Röntgen- oder CT-Bild - der anschließenden Betrachtung und Auswertung am Computer statt am Mikroskop. Darüber hinaus tragen digitale Prozesse im Labor und in der Diagnostik sehr zur Qualität der Arbeit in der Pathologie bei, da Prozesse nachvollziehbar werden und eine entsprechend schnelle und eindeutige Kommunikation möglich wird. Ich freue mich über diesen Prozess der Digitalisierung für die Pathologie und für mich und meine Mannschaft, die dadurch entlastet werden, auch wenn wir bisher nur einen Bruchteil der Möglichkeiten nutzen.

Bley: Die Digitalisierung der Arbeitsprozesse hat schon lange Einzug in die Routine der Radiologie gehalten und ist aus unserem Alltag nicht mehr wegzudenken. Die Zeit der Laserfilm-Befundung am Alternator ist seit Jahren vorbei. Digitale Aufnahmen werden digital betrachtet und ausgewertet, digital archiviert und auch digital in den Konferenzen präsentiert. Dank fortschreitender Softwarelösungen kann auch die Vielzahl der Bildinformationen besser ausgewertet werden. Wenn etwa 3D-Darstellungen benutzt werden, um die Komplexität eines Befundes plastischer zu veranschaulichen, oder aber die zeitliche Dynamik der Durchblutung eines Beines mittels der zeitlich aufgelösten MR-Angiografie untersucht wird. Darüber

hinaus gibt es schon sehr weit fortgeschrittene Bemühungen, mittels computerassistierter Diagnosesysteme die Bildauswertung der Radiologen zu unterstützen. Diese Entwicklungen sind notwendig, da die Vielzahl der Bildinformationen nahezu exponentiell gewachsen ist. So kann es vorkommen, dass etwa in der Computertomografie eines Patienten über 1200 Schichtbilder zu betrachten sind. Ohne digitale Unterstützung wäre das in der täglichen Routine kaum möglich.

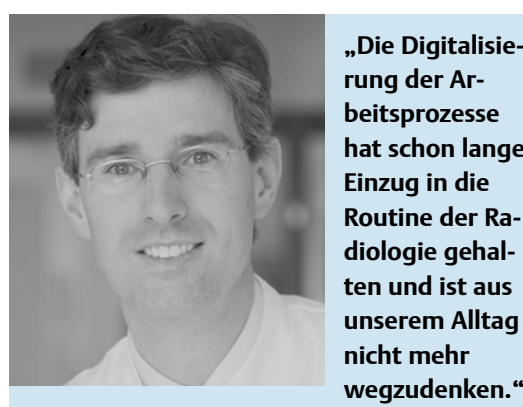

Prof. Dr. Thorsten Bley, Direktor des Instituts für Röntgendiagnostik am Universitätsklinikum Würzburg

? Welche Chancen, aber vielleicht auch Risiken verbinden Sie mit Big Data als einer Möglichkeit, Kausalitätszusammenhänge völlig neu zu evaluieren?

Knüichel-Clarke: Bei aller Vorsicht in der Verwendung des Begriffs „Big Data“ oder „Massendaten“ und in dem Bewusstsein, dass der damit verbundene Inhalt einem ständigen Wandel unterliegt, verbinde ich damit grundsätzlich ein großes Potenzial wie auch eine Entlastung für unsere Berufsgruppen und auch das Individuum. Dabei bin ich mir bewusst, dass zu den 3 klassischen quantifizierbaren „Vs“ der Definition (Volume, Velocity und Variety) die rezent ergänzten „Vs“ - Value und Validity - integraler Bestandteil des Umgangs mit Massendaten sein müssen. Ich bin auch davon überzeugt, dass die sorgfältige Verwaltung von Massendaten unsere eigenen Fähigkeiten überschreiten kann und damit vordergründig positive Weiterentwicklungen möglich werden, mit denen wir uns als Gesellschaft erst noch konstruktiv auseinandersetzen müssen. Hierzu gäbe es noch viel mehr zu sagen, allerdings bin ich auch sehr gespannt darauf, wie die Radiologen als „Verwalter von Massendaten“ denken und damit umgehen werden.
Bley: Für die radiologische Bildgebung ist die Kenntnis von Kausalitätszusammenhängen und Zeichen für die Früherkennung von Erkrankungen von zentraler Bedeutung. Um „Big Data“ für diese Zwecke zu generieren, werden in der sogenannten nationalen Kohorte nicht weniger als 30000 Ganzkörper MRT-Untersuchungen durchgeführt, die die MRT-Bildgebung des Gehirns, des Herzens, des Thorax, des Abdomens und des Skelettsystems umfassen. Das hoch gesteckte Ziel dieser sehr groß angelegten Kohortenstudie ist es, Biomarker abzuleiten, die einen subklinischen Krankheitszustand erkennen lassen und eine gezieltere Primärprävention erlauben.

? Bitte vervollständigen Sie zum Abschluss diese Sätze:

\section{? Pathologie und Radiologie ste-} hen gemeinsam für ...

Knüchel-Clarke: ... interdisziplinär aktive Dienstleitungsfächer und wichtige Forschungstreiber für Gewebe-Diagnostik und Therapiemonitoring.

Bley: ... und übernehmen im klinischen Alltag somit die Funktion des „gatekeepers" für die Therapie und die Behandlungspfade unserer Patienten.

? Die Radiologie kann von der Pathologie lernen, wenn es darum geht..

Bley: ... zu verstehen, welche histologischen bzw. histochemischen Strukturen und Eigenschaften dem charakteristischen Bildeindruck zugrunde liegen.

? Die Pathologie kann von der Radiologie lernen, wenn es darum geht

Knuichel-Clarke: ... zu erfahren wo das Gewebe herkommt, das unter dem Mikroskop liegt.

Frau Professor Knüchel-Clarke, Herr Professor Bley, vielen Dank für das Gespräch! 